\title{
ON TURNBULL IDENTITY FOR SKEW-SYMMETRIC MATRICES
}

\author{
TÔRU UMEDA \\ Department of Mathematics, Faculty of Science, \\ Kyoto University, Kyoto 606-8502, Japan
}

(Received 20 August 1997)

Dedicated to Professor Takeshi Hirai

on the occasion of his 60th birthday

\begin{abstract}
In the last six lines of Turnbull's 1948 paper, he left an enigmatic statement on a Capelii-type identity for skew-symmetric matrix spaces. In the present paper, on Turnbull's suggestion, we show that certain Capelli-type identities hold for this case. Our formulae connect explicitly the central elements in $U\left(\mathfrak{g l}_{n}\right)$ to the invariant differential operators, both of which are expressed with permanent. This also clarifies the meaning of Turnbull's statement from the Lie-theoretic point of view.
\end{abstract}

Keywords: centre of universal enveloping algebra; Capelli identity; permanent

AMS 1991 Mathematics subject classification: Primary 17B35; 15A15

\section{Introduction}

About a half century ago, in the last few lines of [17] treating a Capelli-type identity for symmetric matrices, Turnbull commented very briefly also on a Capelli-type identity for skew-symmetric matrices. Instead of showing its explicit formula, he just suggested that an adaptation could be made to cover the skew-symmetric case by using the permanent with the negative sign diagonal shift. Since then, $[3]$ seems to be the only literature that deals with this identity. In [3], a certain formula is given on the suggestion above, but its proof is left as an exercise of their similar combinatorial proof of the other Turnbull identity for symmetric matrices. Actually, the formula presented in [3] is not the identity for central elements in the universal enveloping algebra $U\left(\mathfrak{g l}_{n}\right)$ of the Lie algebra $\mathfrak{g l}_{n}$, so that it does not give an answer to the Capelli problem in the sense of [5]. Partly for this reason, the authors claimed that Turnbull's identity is less interesting than the skew-symmetric analogue treated by [5] and [7].

In this paper, we will give another candidate to which Turnbull's idea extends. Our formula is for central elements in $U\left(\mathfrak{g l}_{n}\right)$ and the proof will clarify the reason why the permanent fits with this Capelli identity for the skew-symmetric case rather than the determinant. Furthermore, through this investigation, we naturally come up with a family of (non-central) Capelli-type identities which contains the identity given in [3].

We use a method of generating functions. As the determinants appear as the matrix coefficients in exterior algebra, the permanents appear as the matrix coefficients in symmetric algebra. Our computations are, thus, quite parallel to those done in [20]. 


\section{Central elements of permanent type}

First we make some convention on notation. For a multi-index $\alpha=\left(\alpha_{1}, \alpha_{2}, \ldots, \alpha_{n}\right)$ consisting of non-negative integers, we put, as usual,

$$
\alpha !=\alpha_{1} ! \alpha_{2} ! \cdots \alpha_{n} !, \quad|\alpha|=\alpha_{1}+\alpha_{2}+\cdots+\alpha_{n} .
$$

Also, for a fixed set of variables $x=\left(x_{1}, x_{2}, \ldots, x_{n}\right)$, we write $x^{\alpha}=x_{1}^{\alpha_{1}} x_{2}^{\alpha_{2}} \cdots x_{n}^{\alpha_{n}}$. A multi-index $\alpha=\left(\alpha_{1}, \alpha_{2}, \ldots, \alpha_{n}\right)$ can be naturally identified with a non-decreasing sequence $I_{\alpha}$ of $|\alpha|$ integers:

$$
\alpha \longleftrightarrow I_{\alpha}=(\overbrace{1, \ldots, 1}^{\alpha_{1}}, \overbrace{2, \ldots, 2}^{\alpha_{2}}, \ldots, \overbrace{n, \ldots, n}^{\alpha_{n}}) .
$$

We denote by $i_{\alpha}$ the $i$ th component of $I_{\alpha}$, i.e. $i_{\alpha}=k$ if

$$
\sum_{r=1}^{k-1} \alpha_{r}<i \leqslant \sum_{r=1}^{k} \alpha_{r} .
$$

With this notation, we indicate how to pick up the rows or the columns from a fixed matrix according to the multi-index. More specifically, for an $n \times n$ matrix $A$ and for multi-indices $\alpha, \beta$, we denote by $A^{\alpha \beta}$ the $|\alpha| \times|\beta|$ matrix whose $(i, j)$ component is given by $A_{i j}^{\alpha \beta}=A_{i_{\alpha} j_{\beta}}$. Note that if all the components of $\alpha, \beta$ consist of either 0 or 1 , this $A^{\alpha \beta}$ is nothing but the submatrix whose rows and columns are, respectively, determined by $I_{\alpha}$ and $I_{\beta}$.

In this paper, we understand the permanent $\operatorname{Per}(A)$ of an $N \times N$ matrix $A=\left(A_{i j}\right)_{i, j=1}^{N}$ with (possibly) non-commutative entries to be defined as

$$
\operatorname{Per}(A)=\sum_{\sigma \in \mathfrak{S}_{N}} A_{\sigma(1) 1} A_{\sigma(2) 2} \cdots A_{\sigma(N) N} .
$$

A basic observation is that the permanents appear to describe the matrix coefficients of the linear transformation in the space of homogeneous polynomials induced from a linear transformation of the base space. In fact, we have, for $N=|\beta|$,

$$
y^{\beta}=\sum_{|\alpha|=N} \frac{x^{\alpha}}{\alpha !} \operatorname{Per}\left(A^{\alpha \beta}\right)
$$

with $y_{j}=\sum_{i=1}^{n} x_{i} A_{i j}$, because

$$
\begin{aligned}
y^{\beta} & =\sum_{1 \leqslant i_{1}, i_{2}, \ldots, i_{N} \leqslant n} x_{i_{1}} x_{i_{2}} \cdots x_{i_{N}} A_{i_{1} 1_{\beta}} A_{i_{2} 2_{\beta}} \cdots A_{i_{N} N_{\beta}} \\
& =\sum_{|\alpha|=N} \sum_{\sigma \in \mathfrak{S}_{N}} \frac{1}{\alpha !} x_{\sigma(1)_{\alpha}} x_{\sigma(2)_{\alpha}} \cdots x_{\sigma(N))_{\alpha}} A_{\sigma(1)_{\alpha} 1_{\beta}} A_{\sigma(2)_{\alpha} 2_{\beta}} \cdots A_{\sigma(N)_{\alpha} N_{\beta}} \\
& =\sum_{|\alpha|=N} \sum_{\sigma \in \mathfrak{S}_{N}} \frac{x^{\alpha}}{\alpha !} A_{\sigma(1)_{\alpha} 1_{\beta}} A_{\sigma(2)_{\alpha} 2_{\beta}} \cdots A_{\sigma(N)_{\alpha} N_{\beta}} \\
& =\sum_{|\alpha|=N} \frac{x^{\alpha}}{\alpha !} \operatorname{Per}\left(A^{\alpha \beta}\right) .
\end{aligned}
$$


Note that as far as $x_{i}$ and $A_{p q}$ commute, this computation is valid even when $A_{p q}$ are not necessarily commutative.

Remark 2.1. The symmetric group $\mathfrak{S}_{N}$ acts on the set of the $N$-tuples of nonnegative integers $I=\left(i_{1}, i_{2}, \ldots, i_{N}\right)$ with $1 \leqslant i_{k} \leqslant n$ by $\sigma \cdot I=\left(i_{\sigma(1)}, i_{\sigma(2)}, \ldots, i_{\sigma(N)}\right)$. Then each $\mathfrak{S}_{N}$ orbit contains a unique element of the form $I_{\alpha}$ for some multi-index $\alpha$. Note that the order of the stabilizer at $I_{\alpha}$ is $\alpha$ !, so that we have a more reduced expression of the matrix element

$$
\frac{1}{\alpha !} \operatorname{Per}\left(A^{\alpha \beta}\right)=\sum_{\left(i_{1}, i_{2}, \ldots, i_{N}\right) \in \mathfrak{S}_{N} \cdot I_{\alpha}} A_{i_{1} 1_{\beta}} A_{i_{2} 2_{\beta}} \cdots A_{i_{N} N_{\beta}} .
$$

Remark 2.2. The following formula is deduced from (2.1) for the identity matrix $A=\mathbf{1}$.

$$
\operatorname{Per}\left(\mathbf{1}^{\alpha \beta}\right)=\alpha ! \delta_{\alpha \beta}
$$

Also from the property that the permanents appear as the matrix coefficients, we see the chain rule:

$$
\operatorname{Per}\left(C^{\alpha \gamma}\right)=\sum_{|\beta|=N} \frac{1}{\beta !} \operatorname{Per}\left(A^{\alpha \beta}\right) \operatorname{Per}\left(B^{\beta \gamma}\right)
$$

Here, $A, B$ and $C$ are $n \times n$ matrices with commutative entries related as $C=A B$, i.e. $C_{i k}=\sum_{j=1}^{n} A_{i j} B_{j k}$, and we assume $N=|\alpha|=|\gamma|$ for the multi-indices $\alpha$ and $\gamma$.

We now construct certain central elements of the universal enveloping algebra $U\left(\mathfrak{g l}_{n}\right)$ of the Lie algebra $\mathfrak{g l}_{n}$ relating to permanent. Let $E_{i j}$ be the standard basis of the Lie algebra $\mathfrak{g l}_{n}$. We put $E_{i j}(u)=E_{i j}+u \delta_{i j} \in U\left(\mathfrak{g l}_{n}\right)$ with a scalar parameter shift and form the matrices

$$
\boldsymbol{E}=\left(E_{i j}\right)_{i, j=1}^{n}, \quad \boldsymbol{E}(u)=\left(E_{i j}+u \delta_{i j}\right)_{i, j=1}^{n} .
$$

Furthermore, for multi-indices $\alpha, \beta$ with $N=|\alpha|=|\beta|$, we define

$$
\boldsymbol{E}^{\alpha \beta}(u)=\boldsymbol{E}^{\alpha \beta}+\mathbf{1}^{\alpha \beta} \cdot(u-\operatorname{diag}(N-1, N-2, \ldots, 1,0)),
$$

and put $\boldsymbol{E}^{\alpha \beta_{\sharp}}=\boldsymbol{E}^{\boldsymbol{\alpha} \beta}{ }_{\sharp}(0)$. Here 1 stands for the identity matrix of size $n \times n$. By definition, the $(i, j)$ component of $\left.\boldsymbol{E}^{\alpha \beta}, u\right)$ is given by $E_{i_{\alpha} j_{\beta}}+(u-N+j) \delta_{i_{\alpha} j_{\beta}}$. Using these matrices, we define the elements $D_{N}, D_{N}(u) \in U\left(\mathrm{gl}_{n}\right)$, for $N=1,2, \ldots$, by

$$
D_{N}=\sum_{|\alpha|=N} \frac{1}{\alpha !} \operatorname{Per}\left(E^{\alpha \alpha}\right), \quad D_{N}(u)=\sum_{|\alpha|=N} \frac{1}{\alpha !} \operatorname{Per}\left(E^{\alpha \alpha}(u)\right) \text {. }
$$

The following theorem is our first goal, which is due to Nazarov [12] (see also [13] and [10]).

Theorem 2.3. The element $D_{N}(u) \in U\left(\mathfrak{g l}_{n}\right)$ defined above is central in $U\left(\mathfrak{g l}_{n}\right)$. 
Remark 2.4. We have another explicit expression of the element $D_{N}$ as

$$
D_{N}=\frac{1}{N !} \sum_{\sigma \in \mathfrak{S}_{N}} \sum_{1 \leqslant i_{1}, \ldots, i_{N} \leqslant n} \prod_{s}\left(E_{i_{\sigma(s)} i_{s}}-(N-s) \cdot \delta_{i_{\sigma(s)} i_{s}}\right),
$$

where the factors corresponding to the index $s=1, \ldots, N$ are arranged from left to right. The equality (2.7) is a consequence of the relation (2.12) below. Note also that the element $D_{N}$ is the same as that given in [10] by the formula

$$
D_{N}=\frac{1}{N !} \sum_{\sigma \in \mathfrak{S}_{N}} \sum_{1 \leqslant i_{1}, \ldots, i_{N} \leqslant n} \prod_{s}\left(E_{i_{s} i_{\sigma(s)}}-(s-1) \cdot \delta_{i_{s} i_{\sigma(s)}}\right)
$$

where the factors are also arranged from left to right. The difference between (2.7) and (2.8) is in the arrangements and the shift. As in the case of Capelli elements, when the arrangement of the factors for the $E_{i j}$ is changed by the transposition, we need to make a correction by reversing the order in the shift (for the proof of Capelli determinant case, see [6]). We will give the proofs of the equalities (2.7) and (2.8) in Appendix A.

Remark 2.5. The element $D_{N}(u)$ can be expanded in $u$ as

$$
D_{N}(u)=\sum_{r=0}^{N} u^{(r)}\left(\begin{array}{c}
N+n-1 \\
r
\end{array}\right) D_{N-r}
$$

where $u^{(r)}=u(u-1) \cdots(u-r+1)$. The proof of this fact is as follows. Introduce a difference operator $\Delta$ by $\Delta \varphi(u)=\varphi(u+1)-\varphi(u)$. Then we have a Leibniz-like formula

$$
\Delta(\varphi(u) \psi(u))=\Delta \varphi(u) \cdot \psi(u)+\varphi(u+1) \cdot \Delta \psi(u) .
$$

Note that this is valid even when $\varphi(u)$ and $\psi(u)$ are non-commutative. It is easy to see that the Leibniz-like formula applied repeatedly to $D_{N}(u)$, as the permanent is multilinear in the columns, yields the result $\Delta D_{N}(u)=(N+n-1) D_{N-1}(u)$. Then (2.9) follows immediately from this and the formula $\Delta u^{(r)}=r u^{(r-1)}$.

Theorem 2.3 can be looked at in a wider context, especially from the view point of Yangians and $R$-matrices (see, for example, $[\mathbf{1 0}, \mathbf{1 2}, \mathbf{1 3}, \mathbf{1 5}]$ ). However, we will give here a proof of a more elementary nature. For the proof of this theorem, we work in the algebra $\mathbb{C}\left[x_{1}, x_{2}, \ldots, x_{n}\right] \otimes U\left(\mathfrak{g l}_{n}\right)$, where the multiplication is defined so that the two subalgebras $\mathbb{C}\left[x_{1}, x_{2}, \ldots, x_{n}\right]$ and $U\left(\mathfrak{g l}_{n}\right)$ are to commute. Let us consider the elements

$$
\eta_{j}=\sum_{i=1}^{n} x_{i} E_{i j}, \quad \eta_{j}(u)=\eta_{j}+u x_{j}=\sum_{i=1}^{n} x_{i} E_{i j}(u) .
$$

The basic commutation relations of these are given in the following.

Lemma 2.6. For any $1 \leqslant i, j \leqslant n$, we have

$$
\eta_{i}(u-1) \eta_{j}(u)-\eta_{j}(u-1) \eta_{i}(u)=0
$$


Proof. It is sufficient to prove that $\eta_{i}(u) \eta_{j}(u)-\eta_{j}(u) \eta_{i}(u)=x_{i} \eta_{j}(u)-x_{j} \eta_{i}(u)$, and this can be shown by a simple calculation:

$$
\begin{aligned}
{\left[\eta_{i}(u), \eta_{j}(u)\right] } & =\sum_{p, q} x_{p} x_{q}\left[E_{p i}(u), E_{q j}(u)\right]=\sum_{p, q} x_{p} x_{q}\left[E_{p i}, E_{q j}\right] \\
& =\sum_{p, q} x_{p} x_{q}\left(E_{p j} \delta_{i q}-E_{q i} \delta_{j p}\right) \\
& =\sum_{p} x_{p} x_{i} E_{p j}-\sum_{q} x_{j} x_{q} E_{p j} \\
& =x_{i} \eta_{j}-x_{j} \eta_{i}=x_{i} \eta_{j}(u)-x_{j} \eta_{i}(u) .
\end{aligned}
$$

Thus the assertion is proved.

For a multi-index $\alpha=\left(\alpha_{1}, \alpha_{2}, \ldots, \alpha_{n}\right)$ with $N=|\alpha|$, we now define

$$
\eta^{(\alpha)}(u)=\prod_{i=1}^{\vec{N}} \eta_{i_{\alpha}}(u-N+i)=\eta_{1_{\alpha}}(u-N+1) \eta_{2_{\alpha}}(u-N+2) \cdots \eta_{N_{\alpha}}(u) .
$$

The notation $\eta^{(\alpha)}(u)$ here is parallel to $u^{(a)}=u(u-1) \cdots(u-a+1)$. The arrow over the symbol indicates the order of the product, as the factors may not commute. However, in this case, Lemma 2.6 guarantees that

$$
\eta^{(\alpha)}(u)=\eta_{i_{1}}(u-N+1) \eta_{i_{2}}(u-N+2) \cdots \eta_{i_{N}}(u),
$$

for any permutation $\left(i_{1}, i_{2}, \ldots, i_{N}\right) \in \mathfrak{S}_{N} \cdot I_{\alpha}$ of $I_{\alpha}=\left(1_{\alpha}, 2_{\alpha}, \ldots, N_{\alpha}\right)$.

By a computation essentially the same as for the formula (2.1), we see

$$
\eta^{(\beta)}(u)=\sum_{|\alpha|=N} \frac{x^{\alpha}}{\alpha !} \operatorname{Per}\left(E^{\alpha \beta}{ }_{\natural}(u)\right) .
$$

This suggests that $D_{N}(u)$ is defined as the trace of non-commutative linear transform $\left(x^{\alpha}\right)_{\alpha} \mapsto\left(\eta^{(\alpha)}(u)\right)_{\alpha}$. Indeed, the fact that $D_{N}(u)$ is central reflects the invariance of the trace under conjugation (cf. Proposition 3.5 in [15]).

Proof of Theorem 2.3. The adjoint action of $G L_{n}$ on $\mathfrak{g l}_{n}$ extends to an automorphism group action on the enveloping algebra $U\left(\mathfrak{g l}_{n}\right)$. We show that the element $D_{N}(u)$ is invariant under this action. The statement of Theorem 2.3 is then paraphrased as follows. Take any $A=\left(A_{i j}\right) \in G L_{n}$, and write its inverse as $A^{-1}=B=\left(B_{i j}\right)$. From $\boldsymbol{E}=\left(E_{i j}\right)$, we form $A \boldsymbol{E} A^{-1}=\boldsymbol{E}^{*}=\left(E_{i j}^{*}\right)$, so that

$$
E_{i j}^{*}=\sum_{k, \ell=1}^{n} A_{i k} E_{k \ell} B_{\ell j}
$$

Under the algebra automorphism of $U\left(\mathfrak{g l}_{n}\right)$ extending this $E \mapsto E^{*}$, the polynomial

$$
D_{N}(u)=\sum_{|\alpha|=N} \operatorname{Per}\left(E^{\alpha \alpha}:(u)\right)
$$


is transformed to

$$
D_{N}^{*}(u)=\sum_{|\alpha|=N} \operatorname{Per}\left(\boldsymbol{E}^{* \alpha \alpha}(u)\right)
$$

The invariance of $D_{N}(u)$ then amounts to the equality $D_{N}(u)=D_{N}^{*}(u)$.

We write the quantities transformed by this automorphism as

$$
\eta_{j}^{*}(u)=\sum_{i=1}^{n} x_{\alpha}\left(E_{i j}^{*}+u \delta_{i j}\right)
$$

and

$$
\eta^{*(\alpha)}(u)=\prod_{i=1}^{\vec{N}} \eta_{i_{\alpha}}^{*}(u-N+i)=\eta_{1_{\alpha}}^{*}(u-N+1) \eta_{2_{\alpha}}^{*}(u-N+2) \cdots \eta_{N_{\alpha}}^{*}(u)
$$

Then, as (2.13) above, $\operatorname{Per}\left(E^{* \alpha \beta}(u)\right)$ appears as the coefficient of $x^{\alpha} / \alpha$ ! in $\eta^{*(\beta)}(u)$. First, we compute the relation between these $\operatorname{Per}\left(\boldsymbol{E}^{* \alpha \beta_{b}}(u)\right)$ and $\operatorname{Per}\left(\boldsymbol{E}^{\alpha \beta_{b}}(u)\right)$.

Lemma 2.7. We have the following relation

$$
\begin{aligned}
\operatorname{Per}\left(\boldsymbol{E}^{* \nu \gamma_{\natural}}(u)\right) & =\sum_{|\alpha|=|\beta|=N} \frac{1}{\alpha ! \beta !} \operatorname{Per}\left(A^{\nu \alpha}\right) \operatorname{Per}\left(\boldsymbol{E}^{\alpha \beta}(u)\right) \operatorname{Per}\left(B^{\beta \gamma}\right) \\
& =\sum_{|\alpha|=|\beta|=N} \frac{1}{\alpha ! \beta !} \operatorname{Per}\left(\boldsymbol{E}^{\alpha \beta}(u)\right) \operatorname{Per}\left(B^{\beta \gamma}\right) \operatorname{Per}\left(A^{\nu \alpha}\right) .
\end{aligned}
$$

Proof. Introduce the following auxiliary elements:

$$
y_{j}=\sum_{i=1}^{n} x_{i} A_{i j}, \quad \eta_{j}^{\prime}(u)=\sum_{i=1}^{n} y_{i}\left(E_{i j}+u \delta_{i j}\right)
$$

and

$$
\eta^{\prime(\alpha)}(u)=\prod_{i=1}^{\vec{N}} \eta_{i_{\alpha}}^{\prime}(u-N+i)=\eta_{1_{\alpha}}^{\prime}(u-N+1) \eta_{2_{\alpha}}^{\prime}(u-N+2) \cdots \eta_{N_{\alpha}}^{\prime}(u)
$$

Note that the relation (2.12) is valid also for $\eta_{j}^{\prime}(u)$, because $y_{j}$ are commutative. We see the relation of $\eta_{j}^{\prime}(u)$ and $\eta_{j}^{*}(u)$ as

$$
\begin{aligned}
\eta_{j}^{*}(u) & =\sum_{i} x_{i}\left(E_{i j}^{*}+u \delta_{i j}\right)=\sum_{i, k, \ell} x_{i}\left(A_{i k} E_{k \ell} B_{\ell j}+u A_{i k} \delta_{k \ell} B_{\ell j}\right) \\
& =\sum_{i, k, \ell} x_{i} A_{i k}\left(E_{k \ell}+u \delta_{k \ell}\right) B_{\ell j} \\
& =\sum_{k, \ell} y_{k}\left(E_{k \ell}+u \delta_{k \ell}\right) B_{\ell j}=\sum_{\ell} \eta_{\ell}^{\prime}(u) B_{\ell j} .
\end{aligned}
$$


Combining this relation, (2.12), the chain rule (2.4), and (2.1), we see the following computation. We write $u_{i}=u-N+i$ for short below:

$$
\begin{aligned}
\eta^{*(\gamma)}(u) & \eta_{1_{\gamma}}^{*}\left(u_{1}\right) \eta_{2_{\gamma}}^{*}\left(u_{2}\right) \cdots \eta_{N_{\gamma}}^{*}\left(u_{N}\right) \\
= & \sum_{1 \leqslant \ell_{1}, \ell_{2}, \ldots, \ell_{n} \leqslant N} \eta_{\ell_{1}}^{\prime}\left(u_{1}\right) \eta_{\ell_{2}}^{\prime}\left(u_{2}\right) \cdots \eta_{\ell_{N}}^{\prime}\left(u_{N}\right) B_{\ell_{1} 1_{\gamma}} B_{\ell_{2} 2_{\gamma}} \cdots B_{\ell_{N} N_{\gamma}} \\
= & \sum_{|\beta|=N} \sum_{\sigma \in \mathfrak{S}_{N}} \frac{1}{\beta !} \eta_{\sigma(1)_{\beta}}^{\prime}\left(u_{1}\right) \eta_{\sigma(2)_{\beta}}^{\prime}\left(u_{2}\right) \cdots \eta_{\sigma(N)_{\beta}}^{\prime}\left(u_{N}\right) B_{\sigma(1)_{\beta} 1_{\gamma}} B_{\sigma(2)_{\beta} 2_{\gamma}} \cdots B_{\sigma(N)_{\beta} N_{\gamma}} \\
= & \sum_{|\beta|=N} \sum_{\sigma \in \mathfrak{S}_{N}} \frac{1}{\beta !} \eta_{1_{\beta}}^{\prime}\left(u_{1}\right) \eta_{2_{\beta}}^{\prime}\left(u_{2}\right) \cdots \eta_{N_{\beta}}^{\prime}\left(u_{N}\right) B_{\sigma(1)_{\beta} 1_{\gamma}} B_{\sigma(2)_{\beta} 2_{\gamma}} \cdots B_{\sigma(N)_{\beta} N_{\gamma}} \\
= & \sum_{|\beta|=N} \frac{1}{\beta !} \eta^{\prime(\beta)}(u) \sum_{\sigma \in \mathfrak{S}_{N}} B_{\sigma(1)_{\beta} 1_{\gamma}} B_{\sigma(2)_{\beta} 2_{\gamma}} \cdots B_{\sigma(N)_{\beta} N_{\gamma}} \\
= & \sum_{|\beta|=N} \frac{1}{\beta !} \eta^{\prime(\beta)}(u) \operatorname{Per}\left(B^{\beta \gamma}\right) \\
= & \sum_{|\alpha|=|\beta|=N} \frac{1}{\alpha ! \beta !} y^{\alpha} \operatorname{Per}\left(E^{\alpha \beta}(u)\right) \operatorname{Per}\left(B^{\beta \gamma}\right) \\
= & \sum_{|\alpha|=|\beta|=|\nu|=N} \frac{1}{\alpha ! \beta ! \nu !} x^{\nu} \operatorname{Per}\left(A^{\nu \alpha}\right) \operatorname{Per}\left(E^{\alpha \beta}(u)\right) \operatorname{Per}\left(B^{\beta \gamma}\right) .
\end{aligned}
$$

By comparing the coefficient of $x^{\nu}$ in both sides, we obtain Lemma 2.7.

With Lemma 2.7 in hand, we now see by noting the relation (2.3):

$$
\begin{aligned}
D_{N}^{*}(u) & =\sum_{|\nu|=N} \frac{1}{\nu !} \operatorname{Per}\left(\boldsymbol{E}^{* \nu \nu}(u)\right) \\
& =\sum_{|\alpha|=|\beta|=|\nu|=N} \frac{1}{\alpha ! \beta ! \nu !} \operatorname{Per}\left(\boldsymbol{E}^{\alpha \beta}(u)\right) \operatorname{Per}\left(B^{\beta \nu}\right) \operatorname{Per}\left(A^{\nu \alpha}\right) \\
& =\sum_{|\alpha|=|\beta|=N} \frac{1}{\alpha ! \beta !} \operatorname{Per}\left(\boldsymbol{E}^{\alpha \beta_{\natural}}(u)\right) \operatorname{Per}\left(\mathbf{1}_{n}^{\beta \alpha}\right) \\
& =\sum_{|\alpha|=|\beta|=N} \frac{1}{\alpha !} \operatorname{Per}\left(\boldsymbol{E}^{\alpha \beta_{\sharp}}(u)\right) \delta_{\beta \alpha} \\
& =\sum_{|\alpha|=N} \frac{1}{\alpha !} \operatorname{Per}\left(\boldsymbol{E}^{\alpha \alpha}(u)\right)=D_{N}(u) .
\end{aligned}
$$

We have thus proved the assertion of Theorem 2.3 .

Remark 2.8. Taking our Borel subalgebra as the standard upper triangular matrices, we denote by $V_{\lambda}$ the irreducible $\mathfrak{g l}_{n}$-module with the highest weight $\lambda=\left(\lambda_{1}, \lambda_{2}, \ldots, \lambda_{n}\right)$, 
where $\lambda_{1} \geqslant \lambda_{2} \geqslant \cdots \geqslant \lambda_{n}$. The eigenvalue of $D_{N}$ on $V_{\lambda}$ is easy to compute from the expression (2.6):

$$
D_{N} \downarrow_{V_{\lambda}}=\sum_{|\alpha|=N}\left(\lambda_{1}+\alpha_{1}-N\right)^{\left(\alpha_{1}\right)}\left(\lambda_{2}+\alpha_{1}+\alpha_{2}-N\right)^{\left(\alpha_{2}\right)} \cdots\left(\lambda_{n-1}-\alpha_{n}\right)^{\left(\alpha_{n-1}\right)} \lambda_{n}^{\left(\alpha_{n}\right)} .
$$

From this expression, for the polynomial representation with integral highest weight $\lambda=\left(\lambda_{1}, \lambda_{2}, \ldots, \lambda_{n}\right)$ with $\lambda_{1} \geqslant \lambda_{2} \geqslant \cdots \geqslant \lambda_{n} \geqslant 0$, we have the vanishing property that $D_{N} \downarrow_{V_{\lambda}}=0$ if and only if $\lambda_{1}<N$.

The proof of the 'if' part follows from the observation, by the pigeon-hole principle, that each term above

$$
\left(\lambda_{1}+\alpha_{1}-N\right)^{\left(\alpha_{1}\right)}\left(\lambda_{2}+\alpha_{1}+\alpha_{2}-N\right)^{\left(\alpha_{2}\right)} \cdots\left(\lambda_{n-1}-\alpha_{n}\right)^{\left(\alpha_{n-1}\right)} \lambda_{n}^{\left(\alpha_{n}\right)}
$$

vanishes under the condition $N>\lambda_{1} \geqslant \lambda_{2} \geqslant \cdots \geqslant \lambda_{n} \geqslant 0$. Also, the same reasoning implies that for any integral $n$-tuple $\lambda=\left(\lambda_{1}, \lambda_{2}, \ldots, \lambda_{n}\right)$ with $\lambda_{1} \geqslant \lambda_{2} \geqslant \cdots \geqslant \lambda_{n} \geqslant 0$, each term above is non-negative. Then the 'only if' part can be seen from the term $\lambda_{1}^{(N)}$ specialized as $\alpha=(N, 0, \ldots, 0)$.

The computation of the eigenvalues of $D_{N}$ also leads to their relation to the Capelli elements:

$$
\sum_{r=0}^{\min (n, N)}(-)^{r} D_{N-r} C_{r}(N-1)=0 \quad(N=1,2, \ldots) .
$$

Here, the $r$ th Capelli polynomial $C_{r}(\mu)$ is defined through the expansion of the ( $n$ th) Capelli polynomial $C(\lambda)$,

$$
C(\lambda+\mu)=\sum_{r=0}^{n}(-)^{r} \lambda^{(r)} C_{r}(\mu)
$$

and $C(\lambda)=\operatorname{det}(\boldsymbol{E}-\lambda+\operatorname{diag}(n-1, n-2, \ldots, 0))$ is defined by the non-commutative determinant. The above relation between the two series of central elements in $U\left(\mathfrak{g l}_{n}\right)$ is analogous to the fact that the generating functions of elementary symmetric polynomials and complete symmetric polynomials are reciprocal. This suggests that there exists a corresponding cohomology vanishing theorem of non-commutative Koszul complex behind it.

\section{The Capelli-type identities}

Let $\mathrm{Alt}_{n}$ be the space of alternating matrices of size $n \times n$. Then the general linear group $G L_{n}$ acts on $\operatorname{Alt}_{n}$ by $\lambda(g): a \mapsto g a^{t} g\left(g \in G L_{n}, a \in \mathrm{Alt}_{n}\right)$. We denote by $t_{i j}$ $(i<j)$ the standard coordinate functions on $\mathrm{Alt}_{n}$, and use the convention on notation that $t_{j i}=-t_{i j}$ for $j \leqslant i$. The differential operator $\partial / \partial t_{i j}$ is abbreviated as $\partial_{i j}$, and we use the convention $\partial_{j i}=-\partial_{i j}$ too. The infinitesimal action $\lambda$ of $\mathfrak{g l}_{n}$ on the space $\mathcal{P}\left(\mathrm{Alt}_{n}\right)$ of polynomials is given by

$$
\lambda\left(E_{i j}\right)=\sum_{a=1}^{n} t_{a i} \partial_{a j}
$$


Thus, forming the two alternating matrices $T=\left(t_{i j}\right)_{i, j=1}^{n}, \partial=\left(\partial_{i j}\right)_{i, j=1}^{n}$, we see

$$
\lambda(E)={ }^{t} T \partial
$$

Our Capelli identity may be regarded as a non-commutative version of the chain rule (2.4) of permanents. We use the same notation $\lambda$ for the action extended to $U\left(\mathfrak{g l}_{n}\right)$ on $\mathcal{P}\left(\mathrm{Alt}_{n}\right)$.

Theorem 3.1. Under $\lambda$, the central element $D_{N} \in U\left(\mathfrak{g l}_{n}\right)$ is mapped to the invariant differential operator on $\mathrm{Alt}_{n}$ as follows:

$$
\lambda\left(D_{N}\right)=\sum_{|\alpha|=|\beta|=N} \frac{\operatorname{Per}\left(T^{\alpha \beta}\right) \operatorname{Per}\left(\partial^{\alpha \beta}\right)}{\alpha ! \beta !} .
$$

As in the previous section, we will use the method of generating functions. This time we work in an extended algebra $\mathbb{C}\left[x_{1}, x_{2}, \ldots, x_{n}\right] \otimes \mathcal{P} \mathcal{D}\left(\mathrm{Alt}_{n}\right)$, where $\mathcal{P} \mathcal{D}\left(\mathrm{Alt}_{n}\right)$ is the ring of polynomial coefficient differential operators on $\mathrm{Alt}_{n}$.

Let us introduce the following two types of elements:

$$
\xi_{a}=\sum_{i=1}^{n} x_{i} t_{a i} \quad(1 \leqslant a \leqslant n), \quad \zeta_{j}=\sum_{a=1}^{n} \xi_{a} \partial_{a j} \quad(1 \leqslant j \leqslant n) .
$$

Then, from the relation (3.1), we see

$$
\zeta_{j}=\sum_{a=1}^{n} x_{i} \lambda\left(E_{i j}\right)
$$

Since the variables $t_{i j}$ are commutative, the elements $\xi_{a}$ are commutative too. Introducing the elements

$$
\zeta_{j}(u)=\zeta_{j}+u x_{j}=\sum_{a=1}^{n} x_{i} \lambda\left(E_{i j}(u)\right)
$$

we state the fundamental commutation relations between $\xi_{a}$ and $\zeta_{j}(u)$ below.

Lemma 3.2. The following commutation relations hold:

(1) $\left[\partial_{p q}, \xi_{a}\right]=x_{q} \delta_{p a}-x_{p} \delta_{q a}$;

(2) $\zeta_{q} \xi_{a}-\xi_{a} \zeta_{q}=\xi_{a} x_{q} ;$ and

(3) $\zeta_{q}(u-1) \xi_{a}=\xi_{a} \zeta_{q}(u)$.

Proof. Assertion (1) is easy to see by a direct computation:

$$
\left[\partial_{p q}, \xi_{a}\right]=\sum_{i} x_{i}\left[\partial_{p q}, t_{a i}\right]=\sum_{i} x_{i}\left(\delta_{p a} \delta_{q i}-\delta_{p i} \delta_{q a}\right)=x_{q} \delta_{p a}-x_{p} \delta_{q a}
$$


For (2), multiply $\xi_{p}$ from the left on both sides of (1) and sum up with respect to $p$. Then we have

$$
\zeta_{q} \xi_{a}-\xi_{a} \zeta_{q}=\xi_{a} x_{q}-\delta_{q a} \sum_{p} \xi_{p} x_{p}
$$

Here, the last summation vanishes, because

$$
\sum_{p} \xi_{p} x_{p}=\sum_{p, i} x_{i} x_{p} t_{p i}=0
$$

as the sum over all the entries of an alternating matrix. The final assertion (3) follows immediately from (2).

Proof of Theorem 3.1. Let us consider the element

$$
\zeta^{(\alpha)}(u)=\prod_{i=1}^{\vec{N}} \zeta_{i_{\alpha}}(u-N+i)=\zeta_{1_{\alpha}}(u-N+1) \zeta_{2_{\alpha}}(u-N+2) \cdots \zeta_{N_{\alpha}}(u) .
$$

Then, from (3.5) and (2.13), we see, on the one hand,

$$
\zeta^{(\beta)}(u)=\sum_{|\nu|=N} \frac{x^{\nu}}{\nu !} \lambda\left(\operatorname{Per}\left(\boldsymbol{E}^{\nu \beta}(u)\right)\right) .
$$

On the other hand, from the definition (3.3) and Lemma 3.2, we have $\zeta^{(\beta)}(0)$

$$
\begin{aligned}
& =\zeta_{1_{\beta}}(-N+1) \zeta_{2_{\beta}}(-N+2) \cdots \zeta_{N_{\beta}}(0) \\
& =\sum_{1 \leqslant k_{N} \leqslant n} \zeta_{1_{\beta}}(-N+1) \zeta_{2_{\beta}}(-N+2) \cdots \zeta_{(N-1)_{\beta}}(-1) \xi_{k_{N}} \partial_{k_{N} N_{\beta}} \\
& =\sum_{1 \leqslant k_{N} \leqslant n} \xi_{k_{N}} \zeta_{1_{\beta}}(-N+2) \zeta_{2_{\beta}}(-N+3) \cdots \zeta_{(N-1)_{\beta}}(0) \partial_{k_{N} N_{\beta}} \\
& =\sum_{1 \leqslant k_{N-1}, k_{N} \leqslant n} \xi_{k_{N}} \zeta_{1_{\beta}}(-N+2) \zeta_{2_{\beta}}(-N+3) \cdots \zeta_{(N-2)_{\beta}}(-1) \xi_{k_{N-1}} \partial_{k_{N-1}(N-1)_{\beta}} \partial_{k_{N} N_{\beta}} \\
& =\sum_{1 \leqslant k_{N-1}, k_{N} \leqslant n} \xi_{k_{N-1}} \xi_{k_{N}} \zeta_{1_{\beta}}(-N+3) \zeta_{2_{\beta}}(-N+4) \cdots \zeta_{(N-2)_{\beta}}(0) \partial_{k_{N-1}(N-1)_{\beta}} \partial_{k_{N} N_{\beta}} \\
& =\quad \cdots \sum_{1 \leqslant k_{1}, k_{2}, \ldots, k_{N} \leqslant n} \xi_{k_{1}} \xi_{k_{2}} \cdots \xi_{k_{N}} \partial_{k_{1} 1_{\beta}} \partial_{k_{2} 2_{\beta}} \cdots \partial_{k_{N} N_{\beta}} \\
& =\sum_{|\alpha|=N} \sum_{\sigma \in \mathfrak{S}_{N}} \frac{1}{\alpha !} \xi_{\sigma(1)_{\alpha}} \xi_{\sigma(2)_{\alpha}} \cdots \xi_{\sigma(N)_{\alpha}} \partial_{\sigma(1)_{\alpha} 1_{\beta}} \partial_{\sigma(2)_{\alpha} 2_{\beta}} \cdots \partial_{\sigma(N)_{\alpha} N_{\beta}}
\end{aligned}
$$




$$
\begin{aligned}
& =\sum_{|\alpha|=N} \sum_{\sigma \in \mathfrak{S}_{N}} \frac{\xi^{\alpha}}{\alpha !} \partial_{\sigma(1)_{\alpha} 1_{3}} \partial_{\sigma(2)_{\alpha} 2_{3}} \cdots \cdots \partial_{\sigma(N)_{\alpha} N_{3}} \\
& =\sum_{|\alpha|=N} \frac{\xi^{\alpha}}{\alpha !} \operatorname{Per}\left(\partial^{\alpha \beta}\right) \\
& =\sum_{|\alpha|=|\nu|=N} \frac{1}{\alpha ! \nu !} x^{\nu} \operatorname{Per}\left(T^{\alpha \nu}\right) \operatorname{Per}\left(\partial^{\alpha \beta}\right) .
\end{aligned}
$$

Comparing this with (3.8), we see

$$
\lambda\left(\operatorname{Per}\left(E^{\nu \beta}\right)\right)=\sum_{|\alpha|=N} \frac{1}{\alpha !} \operatorname{Per}\left(T^{\alpha \nu}\right) \operatorname{Per}\left(\partial^{\alpha \beta}\right) .
$$

Then, putting $\nu=\beta$ and summing up for all $\beta$ with $|\beta|=N$, we obtain the assertion of Theorem 3.1.

Remark 3.3. In the proof above, we used only the relations (3.1) and Lemma 3.2 (3), so that the same proof can be applied for the action of $G L_{n}$ by the right multiplication on the matrix space $\operatorname{Mat}(m, n)$. For this case, the Capelli identities are obtained by Nazarov [12] (see also [10]). Furthermore, in the proof of Lemma 3.2 (2), we see why the permanent, not the determinant, fits directly with the case of skew-symmetric matrices, while for the action of $G L_{n}$ on the matrix space $\operatorname{Mat}(m, n)$ mentioned above, both types of the Capelli identities using determinants and permanents appear.

Remark 3.4. The anti-symmetric analogue of the Capelli identity obtained in $[\mathbf{3}]$ is the special case of our (3.9) with $N=n$ and $\nu, \beta=(1,2, \ldots, n)$. The formula in [3] seems slightly different from ours: first, a redundant signature sneaked into the formula; second, the authors express their formula in terms of a normal ordering for the differential operators. We give an explanation for the latter point. Let us introduce the notation of the normal ordering : : to indicate the operation to move the partial differential operators, $\partial_{i j}$, to the right and the multiplication operator, $t_{i j}$, to the left. Then, in our notation, the formula in [3] reads as follows:

$$
\lambda\left(\operatorname{Per}\left(\boldsymbol{E}_{t}\right)\right)=: \operatorname{Per}\left({ }^{t} T \partial\right):
$$

This is the resulting formula when we take the permanent of both sides of (3.2). In the symbol of the normal ordering, we may compute as if the operators $\partial_{i j}$ and $t_{i j}$ commute, so that the chain rule (2.4) is formally applied to the expansion of the righthand side of (3.10). We see then that (3.10) is deduced from our (3.9) with $N=n$ and $\nu, \beta=(1,2, \ldots, n)$.

\section{Appendix A.}

Here we give the proofs of the equalities (2.7) and (2.8). We introduce some notation to make a new matrix from an $n \times n$ matrix $A$. For two $N$-tuples of non-negative integers 
$I=\left(i_{1}, i_{2}, \ldots, i_{N}\right), J=\left(j_{1}, j_{2}, \ldots, j_{N}\right)$ with $1 \leqslant i_{k}, j_{k} \leqslant n$, we denote by $A^{I J}$ the $N \times N$ matrix whose $(p, q)$ component is given by $A_{p q}^{I J}=A_{i_{p} j_{q}}$. We denote by $|I|$ the size $N$ of $I$. When $I$ and $J$ consist of non-decreasing sequences of the form $I=I_{\alpha}, J=I_{\beta}$, the matrix $A^{I J}$ coincides with $A^{\alpha \beta}$ introduced in $\S 2$. Similarly as (2.5), we define

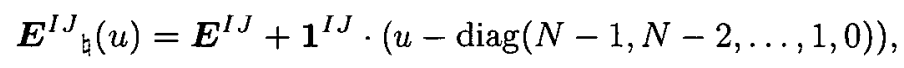

and put $\boldsymbol{E}^{I J_{\natural}}=\boldsymbol{E}^{I J_{\natural}}(0)$. Then the right-hand side of (2.7) can be written as

$$
\frac{1}{N !} \sum_{|I|=N} \operatorname{Per}\left(\boldsymbol{E}^{I I}\right)
$$

To verify (2.7), it thus suffices to show $\operatorname{Per}\left(\boldsymbol{E}^{I I_{\natural}}\right)=\operatorname{Per}\left(\boldsymbol{E}^{I_{\alpha} I_{\alpha}}\right)$ for $I$ in the orbit $\mathfrak{S}_{N} \cdot I_{\alpha}$, as the cardinality of the orbit is $N ! / \alpha !$. This is seen from a more general invariance

$$
\operatorname{Per}\left(\boldsymbol{E}^{\sigma \cdot I \tau \cdot J_{\natural}}(u)\right)=\operatorname{Per}\left(\boldsymbol{E}^{I J}{ }_{\natural}(u)\right),
$$

for $\sigma, \tau \in \mathfrak{S}_{N}$. For the change of rows, it is obvious from the definition of the permanent that $\operatorname{Per}\left(\boldsymbol{E}^{\sigma \cdot I J_{\natural}}(u)\right)=\operatorname{Per}\left(\boldsymbol{E}^{I J_{\natural}}(u)\right)$ for $\sigma \in \mathfrak{S}_{N}$. The invariance under the change of columns follows from (2.12). To see this, we define a quantity similar to (2.11) for $I=\left(i_{1}, i_{2}, \ldots, i_{N}\right)$ by

$$
\eta_{I}^{(N)}(u)=\prod_{s=1}^{\vec{N}} \eta_{i_{s}}(u-N+s)=\eta_{i_{1}}(u-N+1) \eta_{i_{2}}(u-N+2) \cdots \eta_{i_{N}}(u) .
$$

Then, parallel to (2.13), we have the expansion

$$
\eta_{I}^{(N)}(u)=\sum_{|\alpha|=N} \frac{x^{\alpha}}{\alpha !} \operatorname{Per}\left(\boldsymbol{E}^{I_{\alpha} I}(u)\right) .
$$

The equality $(2.12)$ is now rewritten as

$$
\eta_{I}^{(N)}(u)=\eta^{(\beta)}(u)
$$

which holds for any $I \in \mathfrak{S}_{N} \cdot I_{\beta}$. Using (A 3) and (2.13) to compare the coefficients in this equality, we obtain $\operatorname{Per}\left(\boldsymbol{E}^{I_{\alpha} I_{\natural}}(u)\right)=\operatorname{Per}\left(\boldsymbol{E}^{I_{\alpha} I_{\beta}}(u)\right)$. This assures the invariance for columns and, hence, completes the proof of (2.7).

The crucial point for the equality (2.8) lies in finding the necessary change in the permanent expression when the matrix $\boldsymbol{E}$ is transposed. Let us put

$$
{ }^{t} \boldsymbol{E}^{I J}{ }_{\natural^{*}}(u)={ }^{t} \boldsymbol{E}^{I J}+\mathbf{1}^{I J} \cdot(u-\operatorname{diag}(0,1, \ldots, N-2, N-1)),
$$

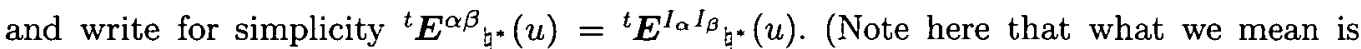
${ }^{t} \boldsymbol{E}^{I J}=\left({ }^{t} \boldsymbol{E}\right)^{I J}$ but not ${ }^{t} \boldsymbol{E}^{I J}={ }^{t}\left(\boldsymbol{E}^{I J}\right)$.) Instead of showing (2.8) itself, we will prove the following:

$$
D_{N}(u)=\sum_{|\alpha|=N} \frac{1}{\alpha !} \operatorname{Per}\left({ }^{t} E^{\alpha \alpha_{\natural^{*}}}(u)\right)
$$


In the course of the proof, this is seen to be equivalent to (2.8). To prove (A 6), we make use of the algebra $\mathbb{C}\left[x_{1}, x_{2}, \ldots, x_{n}, y_{1}, y_{2}, \ldots, y_{n}\right] \otimes U\left(\mathfrak{g l}_{n}\right)$ with doubled formal variables. We put

$$
Z(u)=\sum_{1 \leqslant i, j \leqslant n} x_{i} y_{j} E_{i j}(u)=\sum_{j=1}^{n} y_{j} \eta_{j}(u)=\sum_{i=1}^{n} x_{i} \bar{\eta}_{i}(u)
$$

where

$$
\eta_{j}(u)=\sum_{i=1}^{n} x_{i} E_{i j}(u), \quad \bar{\eta}_{j}(u)=\sum_{i=1}^{n} y_{i} E_{j i}(u) .
$$

Then, by Lemma 2.6 , we have the following commutation relations:

$$
\eta_{i}(u-1) \eta_{j}(u)=\eta_{j}(u-1) \eta_{i}(u), \quad \bar{\eta}_{j}(u) \bar{\eta}_{i}(u-1)=\bar{\eta}_{i}(u) \bar{\eta}_{j}(u-1) .
$$

The former is nothing but Lemma 2.6 itself, and the latter is deduced from the former by the application of the anti-automorphism $E_{i j} \mapsto E_{j i}$ of $U\left(\mathfrak{g l} \mathfrak{l}_{n}\right)$. Let us introduce the counterpart of (A 2) for $\bar{\eta}_{j}(u)$ by

$$
\bar{\eta}_{I}^{(N)}(u)=\prod_{s=1}^{\vec{N}} \bar{\eta}_{i_{s}}(u-s+1)=\bar{\eta}_{i_{1}}(u) \bar{\eta}_{i_{2}}(u-1) \cdots \bar{\eta}_{i_{N}}(u-N+1)
$$

with $I=\left(i_{1}, i_{2}, \ldots, i_{N}\right)$. We will write $\bar{\eta}^{(\alpha)}(u)$ for $\bar{\eta}_{I_{\alpha}}^{(N)}(u)$. Then, by virtue of the symmetry in the commutation relations (A 8), we can translate the identities for the $\eta$ to those for the $\bar{\eta}$. The identity (A 3) is transferred to

$$
\bar{\eta}_{I}^{(N)}(u)=\sum_{|\beta|=N} \frac{y^{\beta}}{\beta !} \operatorname{Per}\left({ }^{t} E^{I_{\beta} I_{\varphi^{*}}}(u)\right) .
$$

Also from (A 4), we have

$$
\bar{\eta}_{I}^{(N)}(u)=\bar{\eta}^{(\alpha)}(u)
$$

for any $I \in \mathfrak{S}_{N} \cdot I_{\alpha}$.

Now consider $Z^{(N)}(u)=Z(u-N+1) Z(u-N+2) \cdots Z(u)$. We expand this in two ways. On the one hand, using the penultimate expression for $Z(u)$ in (A 7$)$, we see from (A4) and (2.13) that

$$
\begin{aligned}
Z^{(N)}(u) & =\sum_{|J|=N} y_{J} \eta_{J}^{(N)}(u) \\
& =\sum_{|\beta|=N} \frac{N !}{\beta !} y^{3} \eta^{(\beta)}(u)=\sum_{|\alpha|=\{\beta \mid=N} \frac{N !}{\alpha ! \beta !} x^{\alpha} y^{\beta} \operatorname{Per}\left(E^{\alpha \beta}(u)\right) .
\end{aligned}
$$

Here, we have used the notation $y_{J}=y_{j_{1}} y_{j_{2}} \cdots y_{j_{N}}$ for $J=\left(j_{1}, j_{2}, \ldots, j_{N}\right)$. On the other hand, use the last expression for $Z(u)$ in (A 7) and reverse the order of multiplication for 
$Z^{(N)}(u)$ as $Z(u) Z(u-1) \cdots Z(u-N+1)$. Then we see from (A 11) and (A 10) that

$$
\begin{aligned}
Z^{(N)}(u) & =\sum_{|I|=N} x_{I} \bar{\eta}_{I}^{(N)}(u) \\
& =\sum_{|\alpha|=N} \frac{N !}{\alpha !} x^{\alpha} \bar{\eta}^{(\alpha)}(u)=\sum_{|\alpha|=|\beta|=N} \frac{N !}{\alpha ! \beta !} x^{\alpha} y^{\beta} \operatorname{Per}\left({ }^{t} E^{\beta \alpha} \dot{-}^{*}(u)\right) .
\end{aligned}
$$

The comparison of the coefficient of $x^{\alpha} y^{\beta}$ in these two expressions of $Z^{(N)}$ tells us the general form of the relation between the permanents of transposed matrices:

$$
\operatorname{Per}\left(\boldsymbol{E}^{\alpha \beta}(u)\right)=\operatorname{Per}\left({ }^{t} \boldsymbol{E}^{\beta \alpha_{q^{*}}}(u)\right) \text {. }
$$

Our assertion (A 6) is immediate from this. From the symmetry between $\eta$ and $\bar{\eta}$ that we have used, the equality (2.8) is also clear.

Remark A 1. The technique of doubling the formal variables is also useful in many aspects (see $[\mathbf{6}]$ ).

\section{References}

1. A. CaPelli, Über die Zurückführung der Cayley'schen Operation $\Omega$ auf gewöhnliche Polar-Operationen, Math. Ann. 29 (1887), 331-338.

2. A. Capelli, Sur les opérations dans la théorie des formes algébriques, Math. Ann. $\mathbf{3 7}$ (1890), 1-37.

3. D. Foata And D. Zeilberger, Combinatorial proofs of Capelli's and Turnbull's identities from classical invariant theory, Electron. J. Combin. 1 (1994), 10pp.

4. R. Howe, Remarks on classical invariant theory, Trans. Am. Math. Soc. 313 (1989), 539-570. (Erratum: Trans. Am. Math. Soc. 318 (1990), 823.)

5. R. HOWE AND T. UMEDA, The Capelli identity, the double commutant theorem, and multiplicity-free actions, Math. Ann. 290 (1991), 565-619.

6. M. ITOH AND T. UMEDA, On central elements in the universal enveloping algebras of the orthogonal Lie algebras, preprint, 1998.

7. B. Kostant and S. Sahi, The Capelli identity, tube domains and the generalized Laplace transform, Adv. Math. 87 (1991), 71-92.

8. J.-L. Koszul, Les algèbre de Lie graduée de type $\mathfrak{s l}(n, 1)$ et l'opérateur de A. Capelli, C. R. Acad. Sci. Paris 292 (1981), 139-141.

9. F. MEYER, Bericht über den gegenwärtingen Stand der Invariantentheorie, Jber. d. Dt. Math.-Verein 1 (1892), 79-292.

10. A. Molev And M. Nazarov, Capelli identities for classical Lie algebras, Math. Ann. 313 (1999), 315-357.

11. A. Molev, M. Nazarov and G. Olshanskit, Yangians and classical Lie algebras, Russ. Math. Surv. 51 (1996), 205-282.

12. M. Nazarov, Quantum Berezinian and the classical Capelli identity, Lett. Math. Phys. 21 (1991), 123-131.

13. M. NAZAROV, Yangians and Capelli identities, in Kirillov's seminar on representation theory (ed. G. I. Olshanski), AMS Translations, Series 2, vol. 181 (1998), pp. 139-163.

14. M. NOUmi, T. UmEDA AND M. WAKaYAma, A quantum analogue of the Capelli identity and an elementary differential calculus on $G L_{q}(n)$, Duke Math. J. 76 (1994), 567-594.

15. A. OKNounkov, Quantum immanants and higher Capelli identities, Transformation Groups 1 (1996), 99-126. 
16. H. W. Turnbull, The theory of determinants, matrices, and invariants (Dover, London, 1960).

17. H. W. Turnbuld, Symmetric determinants and the Cayley and Capelli operators Proc. Edinb. Math. Soc. 8 (1948), 76-86.

18. T. UmEDA, The Capelli identities, a century after, Sugaku 46 (1994), 206-227 (in Japanese). (English translation: Selected Papers on Harmonic Analysis, Groups, and Invariants (ed. K. Nomizu), AMS Translations, Series 2, vol. 183 (1998), pp. 51-78.)

19. T. UMEDA, Newton's formula for $\mathfrak{g l}_{n}$, Proc. Am. Math. Soc. 126 (1998), 3169-3175.

20. T. UMEDA, On the proof of the Capelli identities, preprint, 1997.

21. H. WEYL, The classical groups, their invariants and representations (Princeton University Press, Princeton, NJ, 1946).

22. D.P. Želobenko, Compact Lie groups and their representations, Translations of Mathematical Monographs, vol. 40 (American Mathematical Society, 1973). 